\title{
Efficient Prediction-Based Validation for Document Clustering
}

\author{
Derek Greene and Pádraig Cunningham \\ University of Dublin, Trinity College, \\ Dublin 2, Ireland \\ \{derek.greene, padraig.cunningham\}@cs.tcd.ie
}

\begin{abstract}
Recently, stability-based techniques have emerged as a very promising solution to the problem of cluster validation. An inherent drawback of these approaches is the computational cost of generating and assessing multiple clusterings of the data. In this paper we present an efficient prediction-based validation approach suitable for application to large, high-dimensional datasets such as text corpora. We use kernel clustering to isolate the validation procedure from the original data. Furthermore, we employ a prototype reduction strategy that allows us to work on a reduced kernel matrix, leading to significant computational savings. To ensure that this condensed representation accurately reflects the cluster structures in the data, we propose a density-biased strategy to select the reduced prototypes. This novel validation process is evaluated on real-world text datasets, where it is shown to consistently produce good estimates for the optimal number of clusters.
\end{abstract}

\section{Introduction}

The task of evaluating the output of a clustering algorithm, referred to as cluster validation, is a fundamental problem in unsupervised learning. One common application of validation is in the identification of suitable values for algorithm parameters, such as the optimal number of clusters $\hat{k}$. Internal validation indices, which make assessments based on intrinsic properties of the raw data, have frequently been used for this task in the past [1. However, these tend to be model dependent in the sense that they make assumptions about the structure of clusters in data [2. On the other hand, external validation techniques, which assess the degree to which a clustering corresponds to the "natural classes" in the data, are not directly applicable for parameter selection since external knowledge will typically be unavailable during the clustering process.

Validation techniques based on stability analysis have recently been shown to be particularly effective in determining the optimal number of clusters in data [2. The stability of a clustering model refers to its ability to consistently replicate similar solutions on data originating from the same source. In practice, this involves repeatedly clustering subsamples of the original dataset. A high level of agreement between the clusterings indicates that the model is appropriate for the data. A related approach for estimating $\hat{k}$ was proposed by Tibshirani et al. [3], 
which was motivated by the concept of prediction accuracy in supervised learning. This prediction-based scheme assesses the stability of a clustering model by measuring the degree to which the model allows us to consistently construct a classifier on a training set that will successfully predict the assignment of objects in a clustering of a corresponding test set.

Prototype reduction techniques have been extensively used in supervised learning for tasks involving large datasets. These techniques are concerned with producing a minimal set of objects or prototypes to represent the data, while ensuring that a classifier applied to this set will perform approximately as well as on the original dataset. In the literature, these techniques are generally divided into two categories: prototype selection techniques seek to identify a subset of representative objects from the original data, while prototype extraction techniques involve the creation of an entirely new set of objects. Most work in prototype reduction has focused on supervised learning tasks, although the concept has been used implicitly as part of many clustering algorithms (e.g. 4]). A comprehensive overview of supervised reduction schemes is provided in [5].

A significant drawback of stability-based validation methods is the computational cost of generating and comparing multiple clusterings. Consequently, these methods have rarely been applied to sparse, large-scale datasets such as text corpora. In this paper, we tackle these issues by proposing an efficient predictionbased validation scheme. Our approach makes use of kernel clustering methods so that we no longer need to generate clusterings in the original high-dimensional space. Furthermore, we propose a novel density-biased prototype reduction strategy that allows us to construct a condensed kernel matrix, leading to substantial efficiency improvements. Our evaluation on text data shows that this strategy results in a 16-20 fold speed-up without significantly impacting upon the validation procedure's ability to correctly identify $\hat{k}$. Note that an extended version of this paper is available as a technical report with the same title [6].

\section{Proposed Method}

For small datasets, stability-based validation techniques offer an attractive option for inferring a value for $\hat{k}$. However, as the number of dimensions grows, the time required to repeatedly apply an algorithm such as standard $k$-means will greatly increase. The number of objects $n$ will also be a limiting factor, as a larger value for $n$ will substantially increase the computational cost of the clustering and the stability assessment procedures, which typically run in $O\left(n^{2}\right)$ time or slower. To address these issues, we now present an efficient prediction-based validation method suitable for use on text corpora.

\subsection{Kernel-Based Stability Analysis}

To avoid having to work in the original high-dimensional feature space, we make use of recently proposed kernel clustering methods. A kernel function is usually represented by an $n \times n$ kernel matrix $\mathbf{K}$, where $K_{i j}$ indicates the affinity between 
objects $x_{i}$ and $x_{j}$. The advantage of using kernel methods in the context of stability analysis stems from the fact that, having constructed a single kernel matrix, we may subsequently generate multiple partitions without referring back to the original data. As the standard $k$-means algorithm has commonly been used in both stability analysis and document clustering, we focus here on the use of the corresponding kernelised $k$-means algorithm.

To form the basis for our validation scheme, we choose the prediction-based method proposed by Tibshirani et al. 3] due to its empirical success and computational advantage over other stability analysis methods. The latter benefit derives from the fact that each run of the clustering algorithm works on a sample of $\frac{n}{2}$ objects only. Formally, the validation process involves applying two-fold cross-validation to randomly split the dataset $\mathcal{X}=\left\{x_{1}, \ldots, x_{n}\right\}$ into disjoint training and test sets, denoted $\mathcal{X}_{a}$ and $\mathcal{X}_{b}$ respectively. Both sets are subsequently clustered using kernel $k$-means with random initialisation. A prediction for the assignment of objects in the test set is then produced by assigning each $x_{i} \in \mathcal{X}_{b}$ to the nearest centroid in training clustering. The accuracy of this prediction is assessed by measuring the degree to which it agrees with the original clustering of $\mathcal{X}_{b}$. For this task, we employ an adjusted version of the prediction strength measure described in [3. However, rather than heuristically choosing from among the potential values, we select the value $k$ that leads to the maximum average score over $\tau$ runs. Since prediction strength exhibits a bias toward smaller values of $k$, we employ the widely-used adjustment technique described in [7] to correct for chance agreement.

As discussed in [2], the choice of classifier used to make predictions should complement the clustering algorithm. To "mimic" the assignment behaviour of the kernel $k$-means algorithm, we employ a kernel nearest centroid classifier, such that each object in $\mathcal{X}_{b}$ is classified as being a member of the class represented by the nearest kernel pseudo-centroid in the training clustering. Subsequently, we use corrected prediction strength to evaluate the degree to which the predicted classification agrees with the clustering of $\mathcal{X}_{b}$ as produced by kernel $k$-means.

\subsection{Kernel Reduction}

In the previous section, we described a stability-based validation method suitable for use on high-dimensional data. However, the validation process still requires $\tau$ runs consisting of clustering and prediction assessment phases, which both run in $O\left(\left(\frac{n}{2}\right)^{2}\right)$ time. Clearly, decreasing $n$ will make the process significantly less computationally expensive. Motivated by the large-scale clustering technique described in [4], an intuitive solution is to create a reduced set of $n^{\prime}<n$ objects, upon which the validation procedure may be applied. However, any such reduction must be performed in a way that preserves the structures in the data.

Many supervised prototype reduction approaches process each class separately. As a result, the reduced prototypes will be "meaningful" in the sense that they will represent regions from a single class only. In the absence of class labels, we must rely upon intrinsic properties of the data to ensure that all structures in the data are adequately represented. Unfortunately, text corpora often 
contain unbalanced cluster sizes, which may also differ in their relative densities, making the task particularly problematic. To address these issues, we propose a reduction scheme consisting of two phases. In the first phase, prototype extraction is used to generate a set of candidate prototypes formed from small homogeneous regions of the data. The second phase selects from among these a subset of $n^{\prime}$ prototypes to build a reduced $n^{\prime} \times n^{\prime}$ kernel matrix, denoted by $\mathbf{K}^{\prime}$.

Firstly, we create a set of extracted prototypes $\mathcal{S}=\left\{s_{1}, \ldots, s_{n}\right\}$ in a manner similar to that employed by the supervised BTS reduction scheme [8], where new prototypes are formed by locally combining subsets of the original dataset $\mathcal{X}$. Formally, we define a neighbourhood $\mathcal{N}_{i}$ as a subset of $\mathcal{X}$ consisting of a seed object $x_{i}$ together with its set of $p$ nearest neighbours. A new prototype $s_{i}$ may be constructed from the mean of these $p+1$ objects. Since we wish to work in the kernel-induced space only, we consider $s_{i}$ to be the pseudo-centroid of the subset $\mathcal{N}_{i}$ as calculated from the values in $\mathbf{K}$. We note that, as regions forming cluster structures will normally be locally homogeneous, the majority of the set of neighbours of each object are likely belong to the same cluster as that object. Therefore, prototypes constructed from the centroid of sufficiently small neighbourhoods will generally be representative of a single natural class.

The problem remains of selecting a subset $\mathcal{S}^{\prime}$ of $n^{\prime}$ optimal prototypes from the $n$ possible candidates. A possible solution is to apply unbiased random sampling to choose $\mathcal{S}^{\prime}$. However, this approach has several drawbacks in the context of validation. Ideally, we wish to select a fraction of prototypes from each class that is proportional to the size of that class in the original data. A single random sample from $\mathcal{S}$ is not guaranteed to achieve this. To illustrate this problem, we consider a small subset of the well-known 20 newsgroups collection, consisting of 300 documents from the 'cryptography' group and 150 documents from the 'hockey' group. Figures 1(a) and 1(b) respectively show the block-ordered matrices corresponding to the full kernel matrix and a reduced matrix produced by randomly selecting seed objects. From the latter, it is evident that the smaller 'hockey' class is not adequately represented by the random reduction process. We observe that reduced prototypes chosen in this way frequently fail to produce a true proxy for the dataset, resulting in poor estimations for $\hat{k}$ in the subsequent validation process. In these cases, the failure is often due to poor sampling of smaller clusters or important sub-regions within clusters. While we could run the process multiple times and aggregate the results, the computational cost would typically negate the benefits of performing prototype reduction.

As an alternative, in the second phase of our reduction procedure we employ a density-biased strategy to select $\mathcal{S}^{\prime}$. This procedure has similar goals to existing density-biased sampling techniques (e.g. 9]), but is deterministic and significantly less computationally demanding. Firstly, we define the compactness of a neighbourhood $\mathcal{N}_{a}$ as the average of the pairwise affinities between its members:

$$
C\left(\mathcal{N}_{a}\right)=\frac{\sum_{x_{i}, x_{j} \in \mathcal{N}_{a}} K_{i j}}{\left|\mathcal{N}_{a}\right|^{2}}
$$




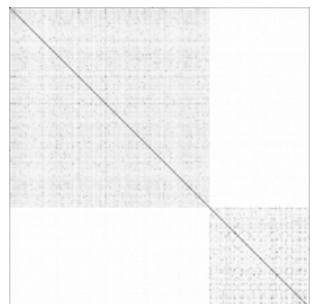

(a)

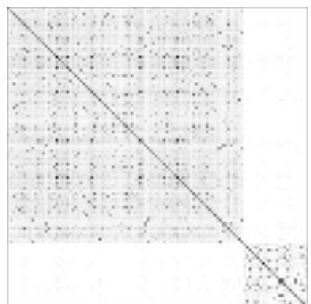

(b)

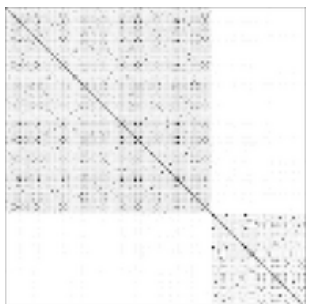

(c)

Fig. 1. Gram matrix for (a) full kernel; (b) kernel reduced by random sampling; (c) kernel reduced by density-biased selection

where $\left|\mathcal{N}_{a}\right|=p+1$. This is equivalent to the "self-similarity" of the pseudocentroid formed from $\mathcal{N}_{a}$. In the selection process, the prototypes in $\mathcal{S}$ are ranked in descending order according to their compactness. From these, we uniformly choose $n^{\prime}=\frac{n}{\rho}$ prototypes, where $\rho$ is the reduction rate that determines the degree to which the number of data objects should be reduced. Specifically, we select every $\rho$-th prototype from the ordered list, thereby ensuring that we represent all density patterns in the data. We then build the reduced kernel matrix $\mathbf{K}^{\prime}$ based on these $n^{\prime}$ prototypes. Rather than computing explicit representations for the new prototypes in the original feature space, we can make use of the values in the original kernel matrix to directly construct $\mathbf{K}^{\prime}$. Formally, the affinity between a pair of reduced prototypes $s_{i}$ and $s_{j}$ is defined as:

$$
K_{i j}^{\prime}=\frac{\sum_{x_{a} \in S_{i}, x_{b} \in S_{j}} K_{a b}}{(p+1)^{2}}
$$

While it is possible that a matrix constructed in this way may not always be positive semi-definite, it has previously been shown in [10] that this does not pose a significant problem for the kernel $k$-means algorithm.

Referring back to our previous example, we see that, unlike the matrix in Figure 1(b), the reduced kernel matrix shown in Figure 1(c) is clearly representative of the two classes in the original dataset. In practice, we consistently observe that this density-biased selection strategy produces a set of extracted prototypes that accurately summarise the underlying structures in the data. We contend that this is due to the inclusion of regions representing clusters of varying densities and all sub-regions within those clusters.

Once we have constructed the reduced kernel matrix, the validation scheme proceeds as described in Section 2.1. The proposed reduction strategy results in a significant decrease in the computational cost of the validation process. Our approach does involve a once-off initialisation step, requiring time $O(n \log n)$ for prototype extraction and $O\left(n^{\prime 2} p^{2}\right)$ for the construction of $\mathbf{K}^{\prime}$. However, the computational gains made in the subsequent validation process are substantial. For each of the $\tau$ runs, the costs associated with clustering and prediction assessment are both reduced to $O\left(\left(\frac{n}{2 \rho}\right)^{2}\right)$. 


\subsection{Application to Document Clustering}

While our proposed method may be used in conjunction with any valid kernel function, for document clustering we make use of a linear kernel that has been normalised according to the approach described in [11. This results in a kernel matrix that is equivalent to that produced by the standard cosine similarity measure. Since this matrix will often suffer from diagonal dominance [10, we address the problem by applying a negative shift to the diagonal of the kernel matrix so as to minimise its trace. A summary of the complete validation process is provided in Figure 2.

\section{Initialisation Phase}

- Construct full $n \times n$ kernel matrix $\mathbf{K}$ using the original data.

- Extract candidate prototypes $\mathcal{S}$, consisting of $n$ neighbourhood pseudo-centroids.

- Evaluate compactness of candidates in $\mathcal{S}$ and sort accordingly in descending order.

- Uniformly select subset $\mathcal{S}^{\prime}$ of $n^{\prime}$ reduced prototypes from the ordered list.

- Construct the $n^{\prime} \times n^{\prime}$ reduced kernel matrix $\mathbf{K}^{\prime}$ based on $\mathcal{S}^{\prime}$ using Eqn. 2

- Apply zero-trace diagonal shift to $\mathbf{K}^{\prime}$.

\section{Validation Phase}

- Produce $\tau$ splits of $\mathcal{S}^{\prime}$ into training and test sets.

- For each value of $k \in\left[k_{\min }, k_{\max }\right]$ :

1. For each split $\left(\mathcal{X}_{a}, \mathcal{X}_{b}\right)$ :

(a) Apply kernel $k$-means to training set $\mathcal{X}_{a}$ using kernel $\mathbf{K}^{\prime}$.

(b) Predict the assignment of documents in $\mathcal{X}_{b}$ based on centroids from clustering of $\mathcal{X}_{a}$.

(c) Apply kernel $k$-means to test set $\mathcal{X}_{b}$ using kernel $\mathbf{K}^{\prime}$.

(d) Evaluate prediction strength and correct for chance.

2. Compute mean corrected prediction strength for $k$.

- Estimate $\hat{k}$ by selecting candidate $k$ with highest mean prediction strength.

Fig. 2. Complete kernel prediction-based validation scheme, with prototype reduction

As mentioned previously, we assume that regions will generally be locally homogeneous, which should be the case when an appropriate kernel function is chosen. To maximise homogeneity, we select a low value for the number of neighbours $(e . g . p=5)$. Empirical evidence suggests that a value of $\rho=4$ for the reduction rate substantially reduces the time required for the validation process, without significantly affecting its accuracy. The selection of $\rho$ is also related to the maximum number of runs $\tau$. The computational gains resulting from prototype reduction facilitate the use of a larger value $(e . g . \tau=200)$ to guarantee the robustness of the overall validation procedure. It must be stressed that, in practice, the use of these general purpose parameter values proved to be effective on a diverse range of datasets, indicating that the proposed validation method is quite robust to the choice of values for these parameters. This allows us to focus on the more immediate task of selecting the number of clusters. 


\section{Empirical Evaluation}

In this section, we evaluate the newly proposed validation scheme on a set of real-world corpora that have previously been used in document clustering. For further details on these corpora and a comprehensive evaluation on artificial data, consult [6]. The experimental process involved applying four predictionbased validation schemes to each corpus across a reasonable range of $k$ values $\left(k_{\min }=2, k_{\max }=10\right)$ and comparing their output with the "true" number of natural classes $\hat{k}$. The first pair of schemes employ the standard $k$-means algorithm with cosine similarity. In the former (KM-S), prediction evaluations are made using prediction strength corrected for chance agreement. In the latter (KM-P), evaluations are made using the partition similarity criterion as described in [12. The second pair of schemes are those introduced in this paper: kernel $k$-means with prediction strength (KKM-S), and kernel $k$-means with prediction strength after prototype reduction (RED-S).

Table 1. Summary of top-3 estimations for $\hat{k}$ on real datasets

\begin{tabular}{|l|c|c|c|c|c|}
\hline Dataset & $\hat{k}$ & KM-S & KM-P & KKM-S & RED-S \\
\hline bbc & 5 & $\mathbf{5 , 4}, 6$ & $\mathbf{5}, 6,7$ & $\mathbf{5}, 6,4$ & $\mathbf{5}, 6,4$ \\
bbcsport & 5 & $4, \mathbf{5}, 3$ & $\mathbf{5}, 6,4$ & $\mathbf{5}, 6,4$ & $\mathbf{5}, 4,6$ \\
classic3 & 3 & $\mathbf{3}, 2,4$ & $\mathbf{3}, 2,4$ & $\mathbf{3}, 4,5$ & $\mathbf{3}, 4,5$ \\
classic & 4 & $3,5,2$ & $3,5,2$ & $5, \mathbf{4}, 2$ & $5, \mathbf{4}, 2$ \\
cstr & 4 & $3,2, \mathbf{4}$ & $3, \mathbf{4}, 2$ & $3, \mathbf{4}, 5$ & $3, \mathbf{4}, 5$ \\
ng17-19 & 3 & $5,4,6$ & $5,4,6$ & $5,4, \mathbf{3}$ & $4,5, \mathbf{3}$ \\
ng3 & 3 & $\mathbf{3}, 4,2$ & $\mathbf{3 , 4}, 5$ & $\mathbf{3 , 4}, 2$ & $\mathbf{3}, 2,4$ \\
reviews & 5 & $2,3,6$ & $2,8,9$ & $2, \mathbf{5}, 4$ & $2,6,3$ \\
\hline
\end{tabular}

Table 1 shows the results of the comparison, indicating the top three estimated values for $\hat{k}$ on the real corpora. In almost all cases, the reduced validation method (RED-S) recommended the same value of $k$ as that chosen when validating based on the full kernel matrix (KKM-S). Only in the case of the reviews dataset, which contains significantly overlapping clusters, did it fail to rate $\hat{k}$ among its top three choices. However, KM-S and KM-P also performed poorly on this corpus. It is interesting to note that both kernel-based techniques consistently outperformed those employing standard $k$-means. In these cases, our evaluations indicate that the application of diagonal dominance reduction prior leads to a non-trivial improvement in validation accuracy.

We observed that, when using kernel-based validation, the application of prototype reduction with $\rho=4$ resulted in a 16-20 fold decrease in the time required for the entire process. For example, on a standard Pentium IV desktop computer, selecting a value for $k$ on the $b b c$ corpus took 55 minutes when using the full kernel matrix, while the same procedure using RED-S took only 3 minutes. In general, RED-S was significantly faster than any of the other strategies considered. 


\section{Conclusion}

We have proposed a practical approach to stability-based validation suitable for the task of estimating the number of clusters in large, high-dimensional datasets such as text corpora. The use of kernel clustering methods allows us to work on a single kernel matrix rather than repeatedly computing distances in the original feature space. Moreover, we have demonstrated that we can significantly decrease the computational demands of the validation process by employing a form of prototype reduction to construct a reduced kernel matrix. Experimental evaluations have shown this validation process to be effective on real-world text corpora, where it consistently produced good estimates for the optimal number of clusters, often outperforming existing methods that are significantly more computationally expensive.

While we have particularly focused on validation in document clustering, we believe that our approach is applicable for a wide variety of other domains and kernel functions, where large datasets would otherwise make stability analysis unfeasible.

\section{References}

1. Jain, A.K., Dubes, R.C.: Algorithms for clustering data. Prentice-Hall, Inc. (1988)

2. Lange, T., Roth, V., Braun, M.L., Buhmann, J.M.: Stability-based validation of clustering solutions. Neural Comput. 16 (2004) 1299-1323

3. Tibshirani, R., Walther, G., Botstein, D., Brown, P.: Cluster validation by prediction strength. Technical Report 2001-21, Stanford University (2001)

4. Cutting, D.R., Pedersen, J.O., Karger, D., Tukey, J.W.: Scatter/gather: A clusterbased approach to browsing large document collections. In: Proc. 15th Annual International ACM/SIGIR Conference. (1992) 318-329

5. Bezdek, J.C., Kuncheva, L.: Nearest prototype classifier designs: An experimental study. International Journal of Intelligent Systems 16 (2001) 1445-1473

6. Greene, D., Cunningham, P.: Efficient prediction-based validation for document clustering. Technical Report CS-2006-22, Trinity College Dublin (2006)

7. Hubert, L.J., Arabie, P.: Comparing partitions. Journal of Classification 2 (1985) $193-218$

8. Hamamoto, Y., Uchimura, S., Tomita, S.: A bootstrap technique for nearest neighbor classifier design. IEEE Tran. Pattern Anal. Machine Intell. 19 (1997) 73-79

9. Palmer, C.R., Faloutsos, C.: Density biased sampling: an improved method for data mining and clustering. In: Proc. 2000 ACM SIGMOD International Conference on Management of Data. (2000) 82-92

10. Greene, D., Cunningham, P.: Practical solutions to the problem of diagonal dominance in kernel document clustering,. In: Proc. 23rd International Conference on Machine Learning. (2006)

11. Schölkopf, B., Smola, A.J.: Learning with Kernels: Support Vector Machines, Regularization, Optimization, and Beyond. MIT Press (2001)

12. Giurcaneanu, C., Tabus, I.: Cluster structure inference based on clustering stability with applications to microarray data analysis. EURASIP Journal on Applied Signal Processing 1 (2004) 64-80 